\title{
Más derecho y menos "justicia". Reflexiones en torno a una aproximación hacia lo jurídico como relación real desde la experiencia de lo percibido
}

\author{
More Law and Less "Justice". Reflections \\ on a Juridic as Aproximation into the Real \\ Relationship since Perceived Experience
}

Fecha de recepción: 3 de junio de 2009

Fecha de aprobación: 23 de septiembre de 2009

EDWIN HORTA*

\begin{abstract}
Resumen
Metodológicamente la cuestión se plantea partiendo de una observación previa: la relación entre percepción y cosa percibida, la cual produce una evidencia que es necesario analizar en sus causas y procedencias. Por este camino se llega a la necesidad de realizar un análisis a la realidad de esta evidencia, por lo que encuentra que el ser personal es una cosa evidente y que por su misma estructura ontológica es sujeto y que en ello, en tal condición de sujeto se encuentra el fundamento mediante el cual le es posible la apropiación de cosas y hacerlas suyas; tal relación de apropiación entre sujeto y cosa suya es real y no mera formulación teórica o de razón. La discusión se plantea en torno a las cosas que pertenecen al sujeto como suyas constituyen el universo de sus derechos, los cuales pueden entrar en conflicto con otros sujetos, conflicto que es necesario resolver, por cuanto permanecer en conflicto no es posible en la configuración de ningún orden social. La solución del conflicto consiste en restituir el orden mediante el reconocimiento de la relación entre el titular del Derecho y sus cosas; a este acto de restitución del Derecho se llama justicia, la cual produce el primer orden de naturaleza social y de especie jurídica. Entonces, se concluye
\end{abstract}

Abogado, Doctor en Filosofía de la Universidad de Navarra, España. Docente Facultad de Derecho de la Universidad Santo Tomás, cátedra filosofía del derecho. Perteneciente al grupo de Investigación Raimundo de Peñafort. 
que el ofuscamiento en la concepción, conceptualización, formulación o aplicación del Derecho deviene en la distorsión de la justicia, por cuanto el objeto específico del acto humano de justicia lo constituye precisamente las cosas que son suyas de las personas. Sus derechos.

\title{
Palabras clave
}

Evidencia, persona, lo jurídico, Derecho, justicia, orden jurídico.

\begin{abstract}
Methodologically, the question arises on the basis of a preliminary observation: it is that the ratio between perception and perceived thing, which produces evidence that is necessary to analyze the causes and origins. Along this road goes down this path Sse the need for an analysis of the reality of eest evidence, so that being encontrándoseuentra staff is one thing clear and that by its very ontological structure is subject and that it, in such condition subject is the basis by which it is possible the appropriation of things and making them his own, that such a relationship between subject and appropriated up to you, is real and not merely theoretical formulation or ratio. This question is discussed about the things that belong to his subject as constituting the universe of their rights, which may conflict with other subjects, it is necessary to resolve conflict, because of remaining in conflict is not possible in the configuration of any social order. Resolving the conflict is to restore order by recognizing the relationship between the owner of dDerecho and things, and in this act of restitution dDerecho is called justice, which produces the first order of species being social and legal. Then he concludes that the blurring in the design, conceptualization, formulation or implementation of dDerecho becomes a distortion of justice, because the subject específico the human act of justice it is precisely the things that are his people. Your rights.
\end{abstract}

\section{Key words}

Evidence, person, being his, relationship, legal, law, justice, legal

\section{INTRODUCCIÓN}

Son muchos los esfuerzos que, incluso antes del siglo XVII a la fecha, se han hecho en torno a una fundamentación, conceptualización, identificación de lo jurídico, bien desde sistemas de pensamiento filosófico, bien desde formulaciones teóricas propuestas al amparo de otras ciencias distintas al Derecho, y nada garantiza que no se puedan formular muchas más en un futuro. Lo que sí es seguro es que disponemos de tantas definiciones del Derecho y tantas "construcciones" de él, que estamos corriendo el riesgo de quedarnos sin él. Desde Kant, cuando afirmaba que todavía andan los juristas corriendo tras una definición del Derecho, a la fecha, es poco lo que hemos adelantado.
Tenemos a nuestra disposición desde fundamentaciones en la naturaleza humana hasta un ordenamiento jurídico sin fundamento alguno, sino en el puro acontecer liberado de cualquier ingrediente metafísico y absolutizado en presupuestos facticos de las índoles más dispares de las que no es posible extraer un principio de seguridad jurídica.

Entre los pensadores del Derecho están: Grocio, Puffendorf, Thomasius, Kant, Fichte, Schelling, Hegel, Bergbohm, Austin, Kelsen, Hart, Dworkin, Kaufmann, Alexy, Reale, Ihering, Del Vecchio, Villey, Vilanova, para citar sólo unos pocos de ellos, sin que dispongamos todavía de una formulación capaz de remontar la actual incertidumbre jurídica. 
Tal incertidumbre se acrecienta aún más cuando entre las propuestas de teorías jurídicas no aparecen con claridad identificados los objetos del conocimiento jurídico, lo que imposibilita disponer de un estatuto epistemológico que nos permita accederlo, entenderlo y aplicarlo. Su ubicación en las regiones del conocimiento ha transitado desde ser una parte de la filosofía, un arte, una ciencia, una técnica a un puro instrumento de operaciones. El Derecho atraviesa una especie de carrera de obstáculos que deben ser salvados y que de no hacerlo correrán hacia un abismo la justicia, la sociedad y todo pretendido orden de convivencia humana será irremediablemente amenazado y precario.

Antes de seguir adelante por este camino, tal vez convenga intentar mirar el Derecho a la luz de otros sistemas de pensamiento filosófico a los acostumbrados desde el siglo XVII, dedicar esfuerzos a identificar sus objetos material y formal, revalorizar sus instituciones fundamentales, su metodología, su sistema de aplicación, definir las relaciones con otros saberes que comparte con él su mismo objeto material, volver a repensar las cuestiones más principales y evidentes, con la esperanza de encontrar por esa vía un panorama menos incierto.

\section{FENÓMENO EXPERIENCIAL}

Hay cosas que han estado siempre ocupando lugares relevantes en el pensamiento humano, cosas que son inherentes a su existencia, cosas que por su intrínseca proximidad a la conciencia humana y a la misma experiencia cotidiana son inevitables a la sensibilidad, a la percepción y a la reflexión del hombre, son aquellas cosas que constituyen predicables exclusivos de los seres capaces de conciencia de sí mismos, de su entorno, de sus acciones $y$, en definitiva, de su propia particular dinámica existencial.

El sentido de la vida, la felicidad, la libertad, el triunfo, el fracaso, la intimidad, las expectativas, la sensación de desposeimiento y muchas otras son esta clase de cosas', dimensiones existenciales, que sólo son posibles que se den en un ser capaz de vivirlas; lo importante de significar aquí no son tanto tales cosas en símismas, sino, precisamente, la razón de ser de tales cosas y el ser que está en capacidad de experimentarlas y del que deviene su sentido (el ser personal).

Para formular más claramente esta dinámica de la condición existencial del hombre podríamos formular las siguientes preguntas: ¿cuál es el fundamento de tales cosas? ¿Cómo tiene que ser un "ser" para que pueda experimentar, es decir, vivir desde sus percepciones y sensaciones, cosas tales (fenómenos) como la felicidad o la infelicidad, la libertad o el ahogamiento, el triunfo o el fracaso, la intimidad y el desposeimiento?

Aunque algo de las respuestas a estas preguntas ya se han anticipado, cuando se dijo que estas dimensiones existenciales (fenómenos) sólo son posibles que se den en un ser capaz de vivirlas a través de su experiencia. Asimismo, con ello se ha mencionado el aspecto psicológico-experiencial, el cual es de importancia definitiva como dato primerísimo del conocimiento, conocimiento sensitivo, imprescindible sí, pero no absoluto ni concluido y, por tanto, se hace necesario abordar los aspectos filosóficos y antropológicos que completen las respuestas a las preguntas formuladas.

La primera cuestión que se plantea al proponer una aproximación a la realidad jurídica y a lo que es específicamente lo jurídico desde la experiencia o lo que es lo mismo, desde lo percibido, a través bien de los sentidos internos o bien externos, es en el fondo una cuestión que hace referencia al modo en que el intelecto humano aprehende las cosas que son y, en consecuencia, la cuestión se

1 El término cosa, en sede filosófica, alberga: desde lo mensurable objetivo hasta lo subjetivo y puramente intramental y llega a abarcar aún lo que es algo en contraposición a lo que no es. Heidegger habla en tres sentidos de la cosa; en sentido restringido, amplio y amplísimo. Para ampliar esta noción véase Heidegger (1964, pp. II ss.). 
ubica necesariamente en el campo de lo filosófico y más concretamente de lo metafísico, toda vez que el conocimiento a este nivel trata o versa sobre el conocimiento del ser-cualquiera que fuere el sistema doctrinal de pensamiento filosófico al que se apele, Realismo o Idealismo-; esto es: el ser es independiente del conocimiento actual que el sujeto tenga de él, para el primero; o que el ser depende absolutamente del sujeto pensante, es decir, que el ser de las cosas es el ser percibido o "construido", para el caso del Idealismo; pero en cualquier caso, como se anotó, es el ser, en sus causas primeras, los objetos del conocimiento filosófico y, por ende, la cuestión se instala en el mundo de lo metacientífico, más allá de lo físico, más allá de lo puramente experimental y no del universo del conocimiento científico estrictamente, puesto que ninguna ciencia considera sus objetos desde el punto de vista del ser; esto no significa de modo alguno que el conocimiento científico y filosófico sean mundos distintos, sino solamente modos y perspectivas diferentes de conocer cosas referentes al ser.

En consecuencia, y para el propósito presente, es decir, la aproximación al ser de la realidad jurídica y su especificidad desde la experiencia sensible, partimos del supuesto de que las dimensiones experiencial y metaexperiencial no son compartimentos estancos, sino que ambas realidades, es decir, la empírica y la del ser, constituyen una sola unidad real y que en punto al conocimiento la existencia sensible no es objeto de una sensibilidad o experiencia aislada del entendimiento, sino que es parte integrante del conocimiento o del saber humano; quien conoce no sólo es el entendimiento aislado ni tampoco la sensibilidad aislada, sino también el hombre sensible con su entendimiento, en definitiva, quien sabe es el hombre y no su entendimiento o sensibilidad y así:

[...] el sujeto cognoscente es tan plenamente real como el objeto conocido que se nos manifiesta como tal en una evidencia primerísima a la que se subordina naturalmente todas las otras: la evidencia de la cosa sensible y visible, de la que debemos partir para juzgar de todas las demás, ya que las demás realidades tienen forzosamente que aparecer en ella. No en vano es ella primum cognitum, es decir, lo primero conocido y en lo que todo se conoce. Y así como el ojo ve todas las cosas bajo el aspecto del color, así el conocimiento humano llega a todo bajo el aspecto de lente entrañado en la naturaleza sensible, como lo manifiesta el hecho de que el hombre no pueda especular sobre los objetos más altos de la metafísica sin la ayuda de las imágenes sensibles, esto es, sin una referencia al ser sensible de donde abstrajo las ideas (Gilson, 1952, pp. 15-16).

No parece posible llegar a un conocimiento del ser, $y$ del que se pueda partir para formular desarrollos ulteriores estables, mediante una ininterrumpida e interminable, a la vez, reflexión sobre lo reflexionado o nueva experiencia sobre lo experimentado ya que en este contexto de proceder "cognitivo" tendríamos cuando más un "ser" siempre provisional; sobre tan "movedizo" e inestable terreno el "conocimiento" obtenido correrá la misma suerte de inestabilidad, ya que lo que en definitiva se compromete en este evento, es el valor mismo del conocimiento, el cual es, precisamente, el ser.

Manser, ha dicho con acierto que "el valor del conocimiento depende de su realidad" (1935, cap. III, S 4, p. 288). Hablar en sede filosófica de la realidad jurídica o de la realidad y especificidad de lo jurídico supone un punto de partida básico y estable que la constituya; en esta vía abundan actualmente las teorías en torno a lo específico y constitutivo radical de lo jurídico, basta con echar una mirada a las teorías de Kelsen, Hart, Dworkin, Kaufmann, Alexi, y muchos otros autores contemporáneos en los cuales se puede advertir claramente que su pretensión de fondo no es otra cosa que definir lo jurídico y la juridicidad. Un recurrente devenir sobre la experiencia o sobre el cogito para extraer de allí la realidad o el ser, sería una tarea indefinida e interminable -por mucho que se quiera 
hablar de un conocimiento siempre haciéndoseen cuyo caso todo se vería reducido a una vana especulación. En consecuencia, pretender hablar por este camino de una realidad jurídica o de un ser de lo jurídico es encaminarse a la aventura de no llegar a nada en concreto, lo cual, en definitiva equivale a confundir la Filosofía del Derecho con un ad infinitum discurso sobre éste.

Quedarse sin el ser es, en definitiva, quedarse sin verdad, "aunque con verdades" de la experiencia o del cogito. El acto del conocimiento filosófico no termina en el enunciado, sino en la cosa misma, pues no se forman enunciados, sino para que con ellos se logre un conocimiento de las cosas -en esta línea el realismo clásico y con principales expositores y tratadistas abunda en argumentos incuestionables.

La incomunicación entre el enunciado y la cosa se produce cuando se afirma que el intelecto sólo conoce la idea, bien sea como esencia o bien sea como actuación del sujeto, así se hace la reflexión del sujeto; el único modo de conocer es convirtiéndose en consecuencia de la certeza de esa reflexión del sujeto en el fundamento de la verdad ${ }^{2}$, asunto muy cuestionable desde un análisis meramente lógico, pues la certeza es un estado de la mente y, por tanto, en sí misma, nada tiene que ver con la realidad por cuanto es

2 "En virtud de esa aberración, de haber centrado el fundamento de la verdad sobre el hecho de ser realización de una posibilidad (tanto si es objetiva como si es subjetiva). Se ha llegado a definir la verdad en primer término como relación, conformidad (adaequatio); y esto ha llevado a la tesis moderna (a partir de Descartes) de la verdad como certeza (Gewissheit). La aberración consiste en haber concebido la filosofía como teoría del conocimiento" (Fabro, s.f., p. 391).

La denuncia de Heidegger, cuando indica el olvido del ser (Vergessenheit des Seins), la causa de los grandes extravíos del pensamiento, ha dado ciertamente en la diana. Nuestra cultura ha ido cayendo del ser en la idea, de la idea en la palabra y de ahí que la recitación; de la contemplación y del testimonio del ser haya ido a parar al decir de lo dicho y al hablar de lo hablado. Se ha olvidado que "el estudio de la filosofía no es para saber qué pensaron los hombres, sino para conocer cuál es la verdad de las cosas", demasiados profesores eruditos han ido declarando fantasías por las candilejas universitarias de este mundo" (Cardona C., 1973, p. 119). perfectamente posible que se pueda estar absolutamente cierto de un error, aunque tal error tenga algunos datos de evidencia, puesto que el error no es conocimiento aunque sea un signo de tal.

Es difícil que desde un sistema de pensamiento filosófico, cuyo propósito sea "darle vida al ser de manera originante" (crear la realidad), se reconozca otro tipo de origen al ser distinto del sujeto y podría ser mucho menos comprensible que ese sistema que "crea" el ser pudiera explicar suficientemente el ser en otro sistema distinto de pensamiento filosófico, pues esto implicaría la imposibilidad de pasar de un sistema a otro, pero sin salirse del sistema mismo.

En esta ocasión no se trata ni de validar, ni de anular ninguno de dichos sistemas de pensamiento filosófico -ni el realismo ni el idealismo-, pero sí de dejar en claro que en ambos sistemas se parte de la evidencia primera, de lo que está ahí: la cosa, el fenómeno, lo que se da y de que en ambos, en tanto sistemas filosóficos, se ha de indagar por la causa fundal o primera de tales evidencias y fenómenos.

\begin{abstract}
No se trata de dar por supuesto que las cosas tienen, por sí mismas, un ser: no es cuestión de aceptar o no un supuesto, sino de admitir o no admitir una evidencia primordial. Tampoco se trata de dar una prueba de la existencia del mundo exterior, porque la inmediatez y evidencia de esta existencia consiste precisamente en ser indemostrable (Gilson, 1952, p. 16).
\end{abstract}

El fin de todo proceso demostrativo es, precisamente, evidenciar algo y no se puede hacer evidente lo que es ya evidente, luego lo ya evidente es precisamente por evidente que no es susceptible de ser demostrado o de ser más demostrado. Lo evidente se muestra, se ve, pero no se demuestra; en cuanto al sujeto se refiere, lo acepta o no lo acepta y lo utiliza como punto de partida del conocimiento, es decir, de indagar por su causa.

La alternativa que se presenta es, en definitiva: 0 la de definir el ser jurídico, la realidad jurídica, en 
términos de producto de pensamiento (absolutizando o desvirtuando el dato de experiencia); o la de definir el ser jurídico, la realidad jurídica, en términos de un ser independiente del pensamiento desde la evidencia experiencial sin absolutizarla o desvirtuarla.

\section{PUNTOS DE PARTIDA EVIDENTES Y EXPERIENCIALES}

Es evidente que existe un ser que se nombra persona y ésta se acepta como tal evidente, con dimensiones de existencia tales como: la vida, la intimidad o posesión de sí, capaz de experiencias, de triunfos, fracasos, libertad, ahogamiento; igualmente, capaz de formular y desarrollar proyectos, un ser con razón y voluntad, mediante cuyo ejercicio se relaciona y coexiste con otros. También, es evidente y se acepta como tal evidencia que ese ser personal tiene y posee cosas, dispone de ellas, las reclama, las exige, dispone del modo de adquirirlas; dichas cosas están en riesgo permanente de perderse; el desconocimiento o violación sobre dichas cosas de la persona trae consecuencias tanto en el orden personal como social; dispone de maneras de protegerlas, de procedimientos para restituirlas; igualmente, existen sistemas para administrarlas en sus relaciones con otros hombres y con el Estado y realidad ocupa un lugar de primerísimo orden en la existencia y experiencia humana, a todo esto es a lo que llamamos realidad jurídica

Ahora bien, estas dos evidencias constituyen materia de conocimiento y, por tanto, existe la posibilidad de una reflexión sobre ellas, las cuales parten desde su dimensión experiencial para llegar a conocer su significación, sus fundamentos y sus causas, a esto es a lo que se llama objeto del conocimiento jurídico.

\section{LA EVIDENCIA DEL SER PERSONAL HUMANO Y SU DIMENSIÓN EXPERIENCIAL}

El anterior acercamiento desde la perspectiva fenomenológica al hombre, en tanto persona, nos proporciona una nota clave de la Antropología, la cual el profesor L. Polo (1991) designa como dual: cuerpo y alma, voluntad e inteligencia, interioridad y medio externo, sujeto y objeto, como las dimensiones antropológicas más radicales y en virtud de las cuales el hombre coexiste con el universo y, a la vez, se sale de él incluyéndolo. Esto significa que evidentemente el hombre no es un producto del universo, ni se agota con él, pero tampoco es una réplica de sí mismo en forma de autoconciencia; por tanto, el hombre no es ser absoluto ni causa de sí mismo, de lo cual se infiere que su ser en sí mismo es un ser dado, recibido, pero recibido como suyo, es decir, como propio, como íntimo. La posesión de su ser, un ser suyo es, precisamente, lo que se contiene en el concepto de intimidad y lo que lo define, en estricto sentido, como persona -el término persona etimológicamente viene de los vocablos per que significa por sí y sonare que significa sonar-. Por tanto, la persona es un ser que suena por sí mismo, que se tiene $y$ se pertenece, $y$ en virtud de dicha posesión puede tener, dar, aportar, identificarse y distinguirse, que son expresiones de su fecundidad y de su peculiar manera de acercarse a la realidad y conocerla ${ }^{3}$.

En esta ocasión no corresponde indagar más profundamente sobre las causas y los fundamentos de esta realidad; por razones del método y por razones del objeto de la reflexión es suficiente con el esclarecimiento de la cuestión que se refiere

3 "todo hombre se pertenece a sí mismo porque es persona [...] la persona tiene estos dos aspectos suyos: la interioridad del espíritu y la exteriorización del cuerpo [...] ambas cosas se compenetran y se complementan: en la interioridad del espíritu se eleva el cuerpo configurando espiritualmente y en exteriorización visible se revela el espíritu encarnado. El hombre es persona en ambos. En ambos se posee soberanamente. Ambas cosas constituyen su radical mismidad, su ser propio, su suun. En la personalidad se funda la autonomía y el dominio del hombre" (Berg L., 1964, p, 108). 
a que el hombre es un ser suyo, no auto hecho y tampoco un ser del mundo. Por cuanto es un ser suyo, se pertenece y se proyecta desde dicha pertenencia, que es, precisamente, la razón por la cual es sujeto, esto es, puede sujetar cosas y tenerlas como suyas. Por cuanto no es del mundo, sino que está en él, se puede apartar de él, lo puede objetivar, dominar y transformar, lo cual es precisamente lo que la persona hace con el mundo de los objetos.

La dinámica de estas notas o características, radicales de la persona humana, expresada en términos de vivencia, como experiencia sensible, se manifiesta en multitud de cosas, tales como: el interrogante por la vida como respuesta al ¿para qué? de la existencia; la felicidad, en tanto posesión de algo que se estima como un bien; la frustración, como el no logro de un resultado propuesto; el éxito como la realización de una meta; el aburrimiento como insatisfacción por lo que hay y aun como insatisfacción derivada de la no aceptación de sí mismo -aburrimiento psicológico y existencial-; la tristeza como desgano del alma, de la fuerza vital; los temores como sentimiento de amenaza de pérdida y la misma intimidad como posesión de sí mismo, tenencia de sí.

Así pues la dimensión experiencial es un accidente que se da en razón no de una pura y exclusiva experiencia aislada, sino en razón de un sujeto que por su condición de ser un ser personal hace posible esta dimensión.

Si observamos con detenimiento estas cosas experienciales que se dan en la persona humana y que, por tanto, constituyen "fenómenos" de su existencia, nos percatamos enseguida que estas cosas no son en sí mismas, sino que son derivadas de la estructura óntica del ser personal. Por cuanto el ser personal se pertenece y se tiene a sí mismo -se sujeta a sí mismo- puede ser sujeto, pero también puede ser desposeído, no en su estructura ontológica, pues ello no es posible a menos que se le elimine; por cuanto se objetiva se puede proyectar y vivenciar el acierto o el fracaso de su propia proyección. En pocas palabras, el estatuto ontológico del ser personal denota un ser con capacidad de dar razón de si precisamente por su dominio sobre sí mismo; dotado de una gran fecundidad vital y cuando tales exigencias de fecundidad no reciben cabal cumplimiento o han sido medianamente satisfechas, se produce como fenómeno experiencial una especie de extrañeza del hombre frente a sí mismo y al mundo; es una forma profunda de inhabitabilidad y desposeimiento.

\section{DIMENSIÓN RELACIONAL DE LA PERSONA HUMANA: EL SER PERSONAL SUJETO Y FUNDAMENTO DE LA RELACIÓN}

Entonces, mientras cada persona posee su singularidad, su ser-en-sí (Insichsein) cada ser personal advierte, en primer lugar, su propia intimidad (ser suyo); en segundo lugar, también advierte la singularidad del otro, en tanto otro y, en consecuencia, su posibilidad relacional con ese otro fundamento de toda comunicación humana (relación de igualdad entre sujetos); y en tercer lugar, advierte su posibilidad de relación con respecto al mundo de los objetos (relación sujeto-objeto), relación de superioridad con respecto al mundo de los objetos.

Este acercamiento que hemos hecho partiendo de la estructura de la persona humana, desde una perspectiva fenomenológica o experiencial, más como un vía de aproximación que como un sistema de proposiciones y verdades formales o razonamientos lógicos o de pensamiento y que nos proporciona unos puntos de reconocimiento comunes, predicables de todas las personas humanas y mientras que en todas ellas se aprecian estos fenómenos comunes, nos permite disponer de un escenario apropiado para inteligir la realidad 
de estos fenómenos y como tales fenómenos indagarlos en su causa fundante o primera.

Siendo coherentes en el proceso experiencial fenomenológico no nos podemos quedar en la mera descripción ni en el análisis de los fenómenos, sino que se ha de llegar a lo que aquellas realidades fenoménicas tienen de contenido real $y$, por tanto, inteligible.

Para ello conviene reparar, una vez más, en la idea central que rige las consideraciones anteriores y es la que el ser personal es un ser en sí mismo y no en otro, ya que si la persona humana no estuviera sustanciada en la ipseidad o mismidad -él mismo (ipse) - no podría -como en efecto sí lo hace- advertir las nociones de despojo, de pérdida o fracaso, de proyección, de relación, de tú y yo, de lo mío y lo tuyo; en pocas palabras, la persona humana es un ser suyo, un ser de sí mismo -dueño de sí que no es lo mismo que decir auto hecho- y es, además, consciente de su dominio y capaz de ejercerlo con respecto a sí, a los demás y a las cosas.

Hasta el momento, la observación adelantada nos permite concluir que la realidad de la persona como que es un ser que se autoposee y se autopertenece, corresponde a una realidad de orden metafísico y no cultural o convencional y, por tanto, se trata de una realidad cuyo fundamento es antropológico y no del orden de las obras de la cultura humana.

Esta evidencia del ser personal, en tanto un ser suyo y con cosas suyas anterior a cualquier fenómeno cultural, es precisamente la que se recoge en casi todas las constituciones políticas modernas, cuando se refieren a los derechos fundamentales y se reconocen tales derechos como anteriores y superiores y, por consiguiente, configuradores de la constitución, advirtiendo con ello que la realidad jurídica tiene un fundamento antropológico y no cultural o político.
Detengámonos ahora un poco en los alcances y en los significados que tiene la autopertenencia y autoposesión, como notas radicales y definitorias de la persona, mediante un procedimiento de análisis.

\section{ESTRUCTURA DE LAS RELACIONES EN EL SER PERSONAL}

Hemos hablado del fundamento de la dimensión relacional de la persona humana, ahora corresponde hablar de la estructura de dicha dimensión relacional. Tenerse a sí mismo o pertenecerse a sí mismo, o poseerse a sí mismo, evidencia tres elementos:

- La existencia de un sujeto o agente que realiza dicha tenencia o pertenencia (sujeto).

- La existencia de la cosa objeto que es tenida o poseída (término).

- La existencia de un tipo de relación que rige el agente o sujeto con la cosa y a ésta con él (el fundamento) que es el que explica, la razón por la cual el sujeto ésta referido al termino y que a la vez tipifica la relación (Berg, 1964, pp. 121 y ss.).

En cuanto al primer elemento, su capacidad de apropiación ha de ser proporcionada a la cosa u objeto tenido, que en este caso es su propio ser -su ser es suyo (la vida es el ser del viviente)-; en consecuencia, quien es capaz de tener su propia existencia por sí mismo, se presenta como sujeto real, distinto a todo lo demás existente y capaz de sujetar, además, otras cosas diferentes a él; y como en tal caso, la cosa tenida es la propia existencia, y siendo ésta la mayor pertenencia posible, podrá, entonces, pertenecerle cosas menores que la existencia -lo que nunca podrá pertenecerle, en ningún caso, será la existencia de otras personas, pues ya esta existencia le pertenece a cada una de ellas, en tanto sujetos de su propia existencia-. Como se ve aquí, la apropiación está dada en su mayor grado, la cual consiste en sujetar la propia existencia. Es por ello que a la persona se le llama 
con propiedad y de manera exclusiva sujeto. Es por ello que sólo el ser personal puede sujetar o tener cosas como suyas y éste es el sentido más radical de la expresión sujeto de derechos, así sólo la persona es sujeto de derechos. Como consecuencia de ello, el Derecho se presenta como una relación real de un ser personal (sujeto) con sus cosas.

En cuanto a la cosa u objeto que se posee o pertenece (segundo elemento) que es el término de la relación y que expresa las cosas que son suyas del sujeto y a las cuales él está ordenado o referido, es necesario reparar si tal cosa inhiere a la propia estructura ontológica del sujeto, en tal caso la cosa constituye un todo con él y, por tanto, compromete su propio ser como es el caso de la intimidad, la libertad o la vida; en caso contrario, vale decir, que la cosa no inhire sustancialmente a la persona su incidencia o compromiso lo es en el orden dinámico u operativo, como es el caso de la tenencia o dominio de cosas materiales.

En cuanto al tercer elemento que es el fundamento de la relación, éste se establece en virtud de la razón por la cual el sujeto (persona) está referido al término (cosa) y que es, en definitiva, lo que causa la relación misma, especificándola, identificándola, caracterizándola. Tenemos que el sujeto está referido a sus cosas, a las cosas que detenta en razón a la pertenencia que el ser personal tiene de sí mismo y que, por tanto, tiene como suyas, y estas cosas suyas, como lo dijimos en la parte anterior, lo son de dos maneras distintas: la primera, si la cosa objeto (término) pertenece al sujeto en virtud de su mismo ser personal como son, por ejemplo, la libertad, la vida, la intimidad, la honra; o si la cosa objeto-término pertenece al sujeto en el orden de sus operaciones o actos, cosas externas a sí mismo, de las cuales él se apropia, las hace suyas, tales como la casa, el trabajo, o el usufructo, como ya se expresó.

Con todo, en ambos casos la relación del sujeto con el término (cosas), es relación de carácter real por cuanto tanto el sujeto que las detenta como las cosas detentadas son distintas entre sí y no meros predicamentos o corolarios lógicos o de pura consideración mental.

Así evidenciamos que el ser personal es en sí mismo un todo relacional que se puede ordenar consigo mismo y con respecto a sí mismo. De hecho, se da el amor a sí mismo o el rechazo de sí mismo, la aprobación o el reproche de sí mismo, el éxito o el fracaso de sí mismo con respecto a su propio proyecto antropológico, que es la objetivación de sí mismo. De igual forma, se da la relación del hombre con sus cosas, con su vida, con su libertad, con su trabajo, con su casa; y se da también la relación del hombre con el hombre a través de dichas cosas.

Tal orden intrínseco que estructura el ser personal consigo mismo y con sus cosas y lo armoniza se puede ver amenazado y de hecho lo está, en el marco de las permanentes relaciones con otros hombres (sujetos) hechos o amenazas que pueden recaer sobre el doble orden de cosas ya dichas, el que ordena su ser personal, como la libertad, la honra, la intimidad o el de las cosas que el hombre ordena hacia sí mismo como su trabajo, la casa, el salario. Esta relación entre el sujeto y lo suyo por cuanto lo suyo es lo que identifica al sujeto es lo que llamamos relación real de especie jurídica.

La persona es lo que tiene de suyo y esto es precisamente lo que la identifica como tal, sustancia individual realmente distinta de otra, y es allí donde hallamos cabalmente el fundamento de lo que se denomina titularidad jurídica, la cual no es otra cosa que aquello por lo cual se identifica al sujeto con lo suyo y, a su vez, a lo suyo con el sujeto (persona). Así el derecho resulta ser un radical de identificación de la persona en relación con otras personas y con sus cosas; es la identificación de estos elementos de la relación sujeto-término lo que subyace en toda decisión judicial, la cual consiste en adjudicar, mediante el esclarecimiento del título (fundamento: 
causa o razón por la cual es suyo) a cada uno lo que se ordena a sí en relación con el otro. A este acto de ordenación de la relación bajo especie jurídica o lo suyo es a lo que se llama propiamente acto de justicia.

Acto real por cuanto el reconocimiento de la libertad, la defensa de la libertad o de la honra, lo mismo que la declaración de la casa o del usufructo como suyos, son ordenes de la realidad evidente y no de simple razón o de pensamiento. Por ello administrar justicia es establecer o, mejor, restablecer el orden de la relación del sujeto con sus cosas en el universo de las relaciones del hombre con otros hombres. La razón por la cual a tal orden se le denomina orden jurídico es porque se produce desde el reconocimiento de lo suyo y no de otros factores como pueden ser los políticos, los ideológicos, los morales o los sociales y así, dicho orden jurídico ordena las conductas de los hombres con respecto a las cosas que son de ellos, al paso que la ley, por ejemplo, puede ordenar también las conductas de los hombres en relación con intereses que se estimen convenientes para la sociedad o el bien común, pero tal orden legal no es en sí mismo orden jurídico, pues -como ya se ha dicho-, éste sólo lo es sino en cuanto lo ordenado es lo referente a las cosas que son suyas, de las personas.

Es necesario reparar que el orden legal en cuanto se refiere a la ordenación de conductas, que se estiman convenientes para la sociedad, el interés colectivo o el bien común, es un orden segundo y de carácter formal o de razón y es formal concretamente por cuanto este orden se establece a la luz de un propósito del legislador que se estima como conveniente o requerido hacia un fin deliberadamente propuesto.

El orden jurídico es un orden primero y de carácter real; es real, puesto que este orden no se establece sobre la base de ninguna pretensión o propósito, sino que se establece con respecto a lo que le corresponde a cada hombre como suyo en sus relaciones con los otros hombres, por tanto, es el orden que se refiere a la convivencia interpersonal, en tanto personas (sustancias individuales), las cuales son cabalmente los sujetos de toda relación. En pocas palabras, no es un orden de un sujeto con respecto a un propósito o fin preestablecido, que es el caso del orden legal (conducta de un sujeto con respecto a un fin), sino sujeto con respecto a otro sujeto (orden jurídico), como ya se ha dicho. Así resulta evidente que sin este orden previo ningún otro orden relacional humano puede subsistir.

Por lo anterior parece claro que el orden jurídico es el fundamento real de todo orden social, en consecuencia, el primer orden se debe establecer para que sea posible cualquier otro orden relacional humano. Por tanto, el orden del Derecho constituye la materia prima para la realización de cualquier otro orden humano bien sea político, social, económico o de cualquier otra especie.

$\mathrm{Al}$ orden resultante del acto de determinar entre las personas lo que se refiere a las cosas suyas o sus derechos, en cuanto cosas que están bajo su dominio, es precisamente a lo que se le llama justicia, así que la justicia es una forma específica de orden y su especificidad es el orden de los derechos y es por esto que la justicia constituye el primer radical social, esto es, la primera base estructural para la configuración del todo social y, como el orden jurídico, resulta de haber ordenado los derechos, al ser el derecho una realidad que inhire a la persona, la justicia es su consecuencia directa y la cual sigue en la misma línea de la realidad del Derecho.

Igual carácter de realidad tiene la injusticia que es la resultante del desorden del derecho en las personas, desorden sobre el cual no es posible ningún orden real social posterior, pues despojar a la persona de su derecho constituye un vaciamiento 
de la persona misma, por tanto, se anula el sujeto real de toda relación social que es la persona. Este vaciamiento equivale, en palabras de Kant, a una verdadera miseria ${ }^{4}$.

La injusticia en el Derecho es una realidad como lo es la justicia y no un ideal o propósito y tal justicia o injusticia consiste en actos humanos. En efecto, si un río se le lleva la casa a un hombre, eso es una desgracia; pero si un hombre le quita la casa a un hombre, eso es una injusticia. Así también se evidencia la realidad de la justicia en el Derecho y que la especie de lo jurídico son las cosas que le pertenecen al hombre en tanto ser personal, lo mismo que se evidencia el carácter de acto humano real que tiene la justicia en el Derecho y también que la materia prima del acto de justicia o de injusticia es el Derecho.

\section{NADIE PUEDE LEGÍTIMAMENTE LLAMAR SUYO LO QUE ES DE OTRO}

Ahora bien, en cuanto el ser personal es sujeto, esto es, ser que se tiene a sí mismo y que como consecuencia de dicha tenencia de sí se deriva la capacidad de tener cosas suyas y advirtiendo que tal ser existe, es decir, que es un ser real, distinto de las cosas a las cuales sujeta y que, por tanto, no se confunde con ellas, de esa manera se establece una relación de superioridad, entre el sujeto y las cosas por él sujetadas; esta superioridad se pone de manifiesto en el uso que las personas hacen de sus cosas, incluyendo la vida misma, su libertad, su cuerpo, su razón y las cosas externas que allega a su dominio. De esto resulta que en las relaciones interpersonales, relación de identidades, pueden quedar comprometidas esas cosas suyas, comprometiendo a la persona misma.

Una persona puede sustituir en el trabajo a otra persona que ha fallecido, en este sentido, lo que

4 "la más grande y repetida forma de miseria a la que estas expuestos los seres humanos consiste más en la injusticia que en la desgracia". Kant, 1925, p. 245). es sustituido es la actividad que realizaba una persona por la actividad que otra persona realiza o va a realizar, pero nunca podrá ser sustituida en sí misma como tal persona, esto es, en sus relaciones familiares, su vida, su mundo afectivo y hasta la misma manera de realizar su trabajo son insustituibles. Por ello la particular configuración ontológica de la persona no permite que ésta pueda ser "transferible, ni componible, ni intercambiable y, por tanto, es insustituible" (Berg, 1964, pp. 12 y ss.).

Precisamente ésta es una estructura antropológica que nos permite ver con toda claridad el fundamento de tres realidades típicamente humanas:

- La realidad de las relaciones que tiene la persona con sus cosas;

- La realidad de las relaciones que tiene la persona con otras personas, y

- La realidad de las relaciones que tienen las personas entre sí en razón a las cosas que cada uno tiene como suyas.

Hemos dicho que dichas relaciones, por cuanto son agenciadas por un ser que se pertenece a sí mismo (dueño de sí o lo que es lo mismo ser persona) comprometen de manera real y afectan ontológica y existencialmente a dicho ser.

En el orden de las relaciones de las personas con sus cosas, bien sea la vida, la libertad, la intimidad, el trabajo, el salario, el arriendo, el automóvil, se trata de cosas, que aunque distintas, como objeto, son suyas con exclusividad; por ello son irrevocablemente suyas a tal punto que otra persona no puede llamarlas suyas; nadie puede Ilamar suyo el automóvil de otro, el salario de otro, el trabajo de otro, la intimidad de otro, la vida de otro, ni apropiárselas, sin que medie consentimiento $y$ que en el evento de dicha apropiación indebida, no consentida, surge el débito como exigencia de restitución, es decir, débito estricta y específicamente jurídico, por cuanto se genera desde 
las cosas que son suyas y por suyas debidas a ellas, configurándose el débito jurídico, el cual es cabalmente lo que constituye la materia de toda exigencia o reclamación en justicia.

Lo anterior quiere decir que la justicia trabaja sobre el presupuesto de que las cosas le pertenecen a alguien, por eso el mundo de lo jurídico es el mundo de las relaciones en el campo de las cosas suyas y de las cosas debidas. Sin este presupuesto real, previo, la justicia sería una voz vacía, una veleidad, una quimera, una mera aspiración subjetiva, individual o colectiva, una bandera política o ideológica, pero, en ningún caso, un objeto de estudio concreto.

\section{LA PREEMINENCIA DEL DERECHO}

Por lo dicho, lo jurídico se refiere de manera específica -fundamental y directamente- al universo de las relaciones, cuya materia u objeto (término) la constituye las cosas que son de alguien y que se concreta en la relación real que tiene un ser personal con sus cosas dentro del marco general de las relaciones interpersonales y no tanto a lo que está normado, establecido -fáctica o positivamente-, regulado, mandado (imperado) o acordado; pues aunque lo establecido, de cualquiera de las maneras enunciadas, como conductas en una sociedad tengan que ver con el mundo de lo jurídico, éstas relaciones de conducta no son en estricto sentido de especie jurídica, sino de naturaleza jurídica ${ }^{5}$ por vía lógica o de razón; por cuanto lo legislado o normado o imperado en o para el mundo de lo jurídico es un producto de la razón humana. La razón puede imperar e inscribir, cualquiera que sea su papel o su propósito, una conducta en el orden de lo jurídico, pero tal inscripción no tiene carácter de fundamento del Derecho.

En otros términos, no es suficiente razón que algo esté mandado o dispuesto a hacerse de una

5 Ya Kant había hecho la distinción en sus principios metafísicos de la teoría del Derecho entre qué es el Derecho y que es de derecho (Kant, 1978, pp. 78 y ss.). determinada manera por la razón humana, para que ese algo sea realmente así por el simple hecho de haber sido mandado; pues el mandato, por sí mismo, por coactivo, acordado, o universal que fuere, no alcanza -en sana lógica- a sustituir o a reemplazar la realidad, si esta realidad imperada ya está dada en la realidad misma, como es el caso del Derecho (lo suyo de las personas), tal mandato no produce más que una realidad "de hecho" (subrealidad o suprarealidad), ya que la realidad de la existencia de la persona y la realidad de la relación que tiene la persona con sus cosas son realidades anteriores y superiores a la realidad del mandato y, en consecuencia, el orden establecido o imperado por vía de razón, fáctico o de fuerza será en todo caso un "orden" mental, lógico, fáctico o de fuerza, pero no un orden realmente jurídico estrictamente considerado.

Por otro lado, toda ciencia tiene sus leyes específicas según el objeto material de su conocimiento y también tienen su propio estatuto epistemológico según su objeto formal; pero, tales leyes no constituyen, dentro de dicha ciencia, más que el dinamismo de ella y en ningún caso, las leyes, de dichas ciencias son por sí mismas su fundamento. Ninguna ciencia es sus leyes. Igual sucede con el Derecho; con respecto a los estatutos material y formal propios de toda ciencia no es en esto una excepción.

La causa por la cual la persona tiene cosas suyas, deriva -como ya se ha dicho- de la misma existencia y constitución particular del ser personal humano y todo acto de imperio, acuerdo, mandato o disposición, será lógicamente en todo caso posterior a la existencia del ser personal y de tipo radicalmente distinto; pues la estructura del ser personal es de tipo ontológico y la estructura del acto de imperio o mandato es de tipo lógico. De lo anterior resulta que la realidad -evidente- de la preexistencia de lo suyo de las personas viene a ser el fundamento primero de lo jurídico. 
Por tanto, buscar el fundamento del Derecho en una realidad distinta al ser personal -nótese que se habla del fundamento de lo jurídico, no de las leyes que lo ponen en movimiento ni del universo en el que se expresa-, como en la sociedad, en la política, en la norma, en el imperio, en lo acordado -participativa o comunicativamente-, es buscarlo donde no existe realmente. En este supuesto de búsqueda se pueden encontrar respuestas que ofrecen universos en los cuales el Derecho se expresa o se manifiesta, pero no donde se fundamenta; puesto que, como ya se anotó, el fundamento es la causa de la relación y no la relación misma; decir, en consecuencia, que la causa del Derecho es lo regulado, lo mandado, lo establecido, cualquiera que sea su modo, es confundir la relación jurídica (relación: sujeto personal, cosa suya) con el fundamento de esta relación, lo cual equivale a desplazar el fundamento -como principio de existencia de la relación-, bien al campo del orden lógico o bien al universo en el que se expresa la relación jurídica, o bien a la manera como se dinamiza o pone en movimiento. Lo anterior, en estricta lógica, equivale en definitiva a una petición de principio, que consiste en pedirle a la relación misma (efecto), o a su expresión o a su dinámica que sea la causa de ella misma (fundamento). La ilicitud lógica de este paso es que se le pide a un efecto o a un acto segundo -como lo es el acto de razón o el mundo de la expresión- que sea el principio de la existencia de una realidad ya existente y por demás evidente.

El fundamento es la causa de la realidad de la relación jurídica, en este caso de la realidad del Derecho, en tanto relación real de un sujeto con lo suyo.

Por lo anterior la justicia de la que trata el Derecho es justicia real, concreta, materializada; un acto posterior, esto es, un acto segundo, que deviene de la preexistencia del Derecho y a él -al Derecho- le debe la justicia su propia existencia como realidad concreta.
En consecuencia, la justicia del Derecho no es un ideal de justicia, ni la que resulta del imperio de lo mandado o establecido, ni tampoco la de un pretendido orden inspirado en "valores", ya que en estos casos obtendríamos, cuando más, órdenes conductuales, órdenes impuestos, órdenes derivados de lo factico, social o político, pero en ningún caso un orden real estrictamente jurídico.

\section{CONCLUSIÓN}

Para obtener de las reflexiones que preceden una conclusión más acabada que contribuya de mejor manera al propósito del presente escrito, resulta de particular conveniencia identificar el concepto relación jurídica desde los elementos que configuran toda relación (Berg, 1964, p. 124). En toda relación se evidencian tres elementos, a saber: Ios sujetos, el término y el fundamento.

El sujeto de la relación es lo que está referido a otro, es decir, el sujeto denota el carácter referencial de la relación. El término es aquello a lo que está referido el sujeto que puede ser: otro sujeto (persona) o una cosa (objeto). El fundamento es la razón causal, el porqué el sujeto está referido al término, explicita el vínculo base entre el sujeto y el término y da cuenta del porqué y del carácter 0 tipo específico de la relación.

Aplicando estos elementos a la realidad de lo jurídico tenemos que el sujeto es todo ser personal (toda persona, ni los animales ni las cosas son sujetos de Derecho). El término, que es aquello a lo que el sujeto está referido son las cosas: la vida, la intimidad, la libertad, el trabajo, el automóvil y todo lo demás que se pueda predicar del sujeto como suyo. Finalmente, el fundamento, que es la causa o el porqué el sujeto está referido al término.

Por tanto, el fundamento es lo que da la existencia a la relación y la explica causalmente, explicitando el porqué de ella; al explicar el porqué un sujeto (persona) tiene cosas que se predican suyas y cosas de las que se puede apropiar. Es evidente 
que tal apropiación no se da en virtud de las cosas mismas -es decir del término-, sino en virtud del sujeto (persona) y, como ya se ha expresado, por cuanto la persona es un ser suyo, dueño de sí, puede, como consecuencia directa derivada de la tenencia de sí mismo, tener cosas que sean suyas. Quien se tiene a sí mismo, persona -que es lo más-, puede tener cosas suyas y disponer de ellas -que es lo menos.

Así se evidencia que es la estructura misma del ser personal y no otra cosa la que hace posible que la persona pueda sujetar cosas que sean suyas (ser sujeto de derechos) y que, por ende, el fundamento del Derecho sea precisamente una consecuencia de la pertenencia que la persona tiene de sí misma y el término son las cosas sobre las cuales recae dicha pertenencia. Dicha relación de la persona con lo suyo es -como lo hemos dicho varias veces- una relación real, es decir, que su existencia -la existencia de tal relación- no se deriva de ningún acto formal o de razón ni tampoco se deriva de otras obras de la cultura.

Trasladando la anterior estructura relacional descrita, a lo que se adelanta en un proceso jurídico para producir una sentencia es concretamente: ubicar el término de la relación jurídica, es decir, las cosas (lo suyo) en el respectivo sujeto (titular) y que esta adjudicación se hace con base en las razones -argumentos con sus respectivos soportes probatorios (fundamento)- que el sujeto aporta para reclamar la pertenencia de sus cosas. Al resultado de este procedimiento de ubicación u ordenación de las cosas (término) en sus respectivos sujetos (titulares) es a lo que se llama acto de justicia.

Por lo dicho queda claro que la justicia del Derecho consiste concretamente en un orden real del sujeto (persona) con respecto a las cosas (término) y con fundamento en la prueba o demostración del porqué el sujeto (o titular) reclama o demanda las cosas como suyas; de allí que la resultante final de este proceder sea el orden de las cosas suyas -orden de los derechos en las personas- y que a este orden se le llame orden jurídico y no orden legal, ni orden social, ni orden político, ni orden moral, puesto que dichos órdenes son subsiguientes al orden jurídico y no pueden subsistir sin el primero.

La dinámica de lo anteriormente descrito se esclarece más aún cuando observamos que las conductas de las personas pueden violentar o, de hecho, violentan o amenazan las cosas que son de otras personas -naturales o jurídicas-; tal violencia o amenaza genera la reacción de los sujetos (titulares) de dichas cosas, reacción que se concreta en una acción de petición o demanda de respeto, reclamo, restitución o reparación ante quien administra justicia (juez), aportando en dicha acción las razones que le asisten para sus demandas (fundamentos, pruebas) de la lesión o violencia ejercida sobre su derecho. El juez inicia el proceso para fundamentar su decisión sobre la base de las pruebas allegadas a él, el sentido de la actividad probatoria es evidenciar la realidad tanto del Derecho como de su lesión y sobre dicha evidencia o demostración funda su providencia final, persiguiendo con ella la reparación o restitución del Derecho en cabeza de su respectivo titular (sujeto) y revertir de esta manera el orden violentado; orden de la personas con respecto a sus derechos. El orden jurídico.

\section{Una anotación final}

Como todo acto humano, el acto de justicia puede estar teñido de intereses, pasiones, compromisos, presiones, influencias ideológicas, errores y es además causa de efectos colaterales. De allí que quien administra justicia deberá adelantar tal acto, de forma que sin descuidar ni pretermitir lo que es el objeto propio de ésta (el reconocimiento del Derecho), habrá de realizarlo desde una posición intelectual que le permita apreciar la realidad en todas sus dimensiones y saber con claridad las circunstancias concretas en las cuales ha de tener lugar su decisión; en pocas palabras, no le basta 
al juez ser un simple operador de la ley ni del proceson sino que debe ser prudente ${ }^{6}$.

La prudencia hace parte del estatuto epistemológico del Derecho e integra el universo del conocimiento jurídico (Hervada, s.f., pp. 606 y ss.).

En la moderna concepción del juez como simple operario u operador subyace, al parecer, un vaciamiento del acto humano de justicia y un ofuscamiento en la concepción misma de lo que es el Derecho. El error, la imprecisión, en la concepción, conceptualización, administración o en el estatuto epistemológico del Derecho, se trasfiere necesariamente al acto de justicia, por cuanto el Derecho, por lo que hemos dicho, es la materia prima con la que se realiza este acto, dejando el acto de justica sin materia prima, esto es sin fundamento real, en una pura y simple expresión sin contenido alguno.

En el comentario al libro del poderoso de Tomás de Aquino se lee: "la corrupción de la justicia tiene dos causas: la falsa prudencia del sabio y la violencia del poderoso".

\section{REFERENCIAS}

Berg, L. (1964). Ética social. Madrid: Rial.

Cardona, C. (Sin fecha). Metafísica de la opción intelectual. Madrid: E Rialp.
Das Wesen des Thomismus, cap. III, S 4, Friburgo, Suiza, 1935, pag. 288.

Gilson, E. (1952). El realismo metódico (estudio preliminar de Leopoldo Palacios, 2a Ed.). Madrid: Rial.

Gilson, E. (2007). La filosofía en la Edad Media. Madrid: Gredos.

Hervada, J. (2005). ¿Qué es el derecho? Bogotá: Temis.

Hervada, J. (Sin fecha). Lecciones propedéuticas de filosofía del derecho. Pamplona, España: Eunsa, Ediciones Universidad de Navarra.

Kant, E. (1978). Introducción a la Teoría del derecho. Madrid: Centro de Estudios Constitucionales.

Pieper, J. (Sin fecha). Las virtudes fundamentales. Madrid: Rialp.

Polo, L. (1991). Inmanencia y trascendencia. Actas de las XXV reuniones filosóficas de la Universidad De Navarra (Vol. 1). Pamplona: Servicio de Publicaciones Universidad De Navarra.

Villey, M. (1979). Compendio de Filosofía del Derecho. Pamplona: Eunsa.

6 La prudencia exige no solamente la clara conciencia o conocimiento de los principios que rigen el objeto de los actos, sino también una muy fina conciencia de la situación concreta en las que éstos van a acontecer. Para ampliar el estudio sobre la prudencia véase a J. Pieper J., Las virtudes fundamentales. 